\title{
Diagnostic Accuracy of First and Early Second Trimester Multiple Biomarkers for Prediction of Gestational Diabetes Mellitus: A Multivariate Longitudinal Approach
}

\section{Elham Shaarbaf Eidgahi}

Shahid Beheshti University of Medical Sciences

Malihe Nasiri

Shahid Beheshti University of Medical Sciences

Nourossadat Kariman

Shahid Beheshti University of Medical Sciences

Nastaran Safavi Ardebili

Islamic Azad University

Masoud Salehi

Iran University of Medical Sciences

Maryam Kazemi

Shahid Beheshti University of Medical Sciences

Farid Zayeri ( $\nabla$ f_zayeri@sbmu.ac.ir)

Shahid Beheshti University of Medical Sciences

\section{Research Article}

Keywords: Gestational diabetes mellitus, Hemoglobin, hematocrit, Fasting blood sugar, Red blood cell count, Diagnostic accuracy

Posted Date: May 13th, 2021

DOI: https://doi.org/10.21203/rs.3.rs-491430/v1

License: (c) (i) This work is licensed under a Creative Commons Attribution 4.0 International License. Read Full License 


\section{Abstract \\ Background}

Gestational Diabetes Mellitus (GDM) is an underlying cause of maternal and newborn morbidity and mortality all around the world. Timely diagnosis of GDM plays an important role in reducing its adverse consequences and burden. This study aimed to determine diagnostic accuracy of multiple indicators in complete blood count (CBC) test for early prediction of GDM.

\section{Methods}

In this prospective cohort study, the data from 600 pregnant women was analyzed. In the study sample, the two-step approach was utilized for the diagnosis of GDM at 24-28 weeks of gestation. We also used the repeated measures of hemoglobin $(\mathrm{Hb})$, hematocrit (Hct), fasting blood sugar (FBS) and red blood cell count (RBC) in the first and early second trimesters of pregnancy as the longitudinal multiple indicators for early diagnosis of GDM. The classification of pregnant women to GDM and non-GDM groups was performed using a statistical technique based on the random-effects modeling framework.

\section{Results}

Among the sample, 49 women (8.2\%) were diagnosed with GDM. In the first and early second trimester of pregnancy, the mean $\mathrm{HcT}, \mathrm{Hb}$ and FBS of women with GDM was significantly higher than non-GDMs $(\mathrm{P}<$ 0.001). The concurrent use of multiple longitudinal data from $\mathrm{HcT}, \mathrm{Hb}, \mathrm{RBC}$ and FBS in the first and early second trimester of pregnancy resulted in a sensitivity, specificity and area under the curve (AUC) of $87 \%$, $70 \%$ and $83 \%$, respectively, for early prediction of GDM.

\section{Conclusions}

In general, our findings showed that the concurrent use of repeated measures data on $\mathrm{Hct}, \mathrm{Hb}, \mathrm{FBS}$ and RBC in the first and early second trimester of pregnancy might be utilized as an acceptable tool to predict GDM in the earlier stages.

\section{Introduction}

Gestational Diabetes Mellitus (GDM) is one of the most common medical complications of pregnancy with considerable increasing prevalence in some parts of the world within the previous decades, especially in developing countries (1-3). It mainly refers to any degree of glucose intolerance with onset or first diagnosis during pregnancy $(1,4)$. Although GDM is mostly symptom-free, it might be an underlying cause of acute or long-term consequences both in mothers and babies. Compared to nonGDM women, mothers with GDM have an increased risk of pre-eclampsia, mental disorders like 
depression, urinary tract infections, type II diabetes and cardiovascular disease (5-8). In addition, untreated GDM may result in some health problems in newborns such as congenital abnormalities, macrosomia, having low blood sugar and icterus. Later in life, babies born from GDM women are at risk of being overweight or obese and developing type II diabetes $(5,9-10)$.

Up to the present time, there has been no globally accepted gold standard for diagnosing GDM. Depending on the diagnostic criteria, researchers have reported a wide range of prevalence rates for GDM in different parts of the world. For example, a comprehensive systematic review and meta-analysis study showed that the Middle East and North Africa had the highest and Europe had the lowest prevalence rate of GDM in the recent years (with the estimated median of $12.9 \%$ and $5.8 \%$, respectively). In this article, the reported range of GDM prevalence was from $1.8 \%$ in Ireland to $25.1 \%$ in Singapore (11). In 2006, the pooled estimates from different criteria showed that GDM affects around 7 percent of expectant mothers in the entire world (12). On the other hand, the International Diabetes Federation (IDF) has estimated a global prevalence of 14\% for GDM in 2013 (13). The estimates also show that GDM imposes a huge economic burden on the health systems of the world countries (14-15).

Regarding adverse outcomes of GDM and its remarkable global burden, detection and management of this disease seems to be crucial for the health of mothers and babies. To date, a variety of screening and diagnostic procedures have been utilized for detecting mothers with GDM and these procedures may vary by country, region, and year. For instance, the American Diabetes Association (ADA) and the World Health Organization (WHO) have recommended different criteria for the diagnosis of GDM in pregnant women (16-19). Apparently, these criteria may lead to different diagnostic power indices (sensitivity, specificity, positive and negative predictive values, area under the ROC curve and so on) subject to the characteristics of the target population $(11,20)$. In addition to the standard methods of screening, a number of studies evaluated the diagnostic accuracy of the first or second-trimester maternal markers for early detection of GDM using different statistical techniques (21-28).

Reviewing the related published literature indicates that most of researchers have investigated the information from a single biomarker or multiple biomarkers in the first or early second trimester of pregnancy for early detection of GDM. The beta-human chorionic gonadotropin ( $\beta$-hCG), tumor-necrosis factor alpha (TNFa), unconjugated estriol (uE3), alfa-fetoprotein (AFP), pregnancy-associated plasma protein A (PAPP-A), soluble endoglin, placental protein 13 (PP13), adiponectin (Adipo), and a number of other adipose tissue-derived and placenta-related factors are among these biomarkers (21-26). At the same time, a number of studies have focused on the available data from the more common indicators in complete blood count (CBC) tests for early prediction of GDM (27-28).

To the best of our knowledge, all of the published researches have used cross-sectional data on a single biomarker or multiple biomarkers in the first or second trimester of pregnancy for early detection of GDM. However, these biomarkers are repeatedly (longitudinally) available from the routine blood tests for women in different trimesters of pregnancy. Regarding this, we conducted the present study to use multiple repeated measures (multivariate longitudinal) data from four routine indicators in CBC test for 
early prediction of GDM. To do this, we applied more advanced statistical techniques to exploit the additional information in repeated measures data from these markers in the first and early second trimester of pregnancy and estimate the predictive accuracy indices for these repeated multiple indicators.

\section{Methods}

\section{Sample under the study}

In this prospective cohort study, 700 pregnant women who consecutively referred to the prenatal clinic of Milad Hospital in Tehran, Capital of Iran, were recruited. The study protocol was approved by the Ethics Committee of Shahid Beheshti University of Medical Sciences and the written informed consent was obtained from all the sample women. At the first step, demographic information and midwifery history of these women were collected by face-to-face interview and the mothers' weight and height was measured using a standard scale and a meter attached to the scale.

The inclusion criteria were singleton pregnancy, gestational age of 13 weeks or less (using a sonography report during the first trimester), maternal age between 18 and 35 years, parity of 3 or less, lack of any diagnosed systemic diseases (including diabetes, chronic hypertension and cardiovascular disease, chronic renal disease, gastroenterology disease, thyroid, epilepsy, hemoglobinopathies and mental disorders), lack of any history of smoking, alcohol use, non-routine drugs use during the present pregnancy, and any history of preeclampsia in pervious pregnancy. In addition, presence of fetal anomalies, polyhydramnios, oligohydramnios, placenta prevail, abruption placenta, abortion, and stillbirth were considered as the exclusion criteria of the present study. Regarding these criteria, 100 subjects were excluded from the study.

\section{Main outcome and biomarkers under the study}

In this research, the main under the study was the presence of GDM at 24-28 weeks of gestation. The twostep approach was used for the diagnosis of GDM in the described sample. In the first step of this approach, we performed a 50-g glucose load test (GLT) and measured the plasma glucose at 1 hour after test. In the second step, we performed a 100-g oral glucose tolerance test (OGTT) for women with the plasma glucose level of $140 \mathrm{mg} / \mathrm{dL}$ or more measured in the first step. Finally, the diagnosis of GDM was made according to the Carpenter and Coustan (C\&C) criteria when at least two of the following four plasma glucose levels were met; fasting level of at least $95 \mathrm{mg} / \mathrm{dl}, 1$ hour level of at least $180 \mathrm{mg} / \mathrm{dl}, 2$ hour level of at least $155 \mathrm{mg} / \mathrm{dl}$ and 3 hour level of at least $140 \mathrm{mg} / \mathrm{dl}$.

For 600 pregnant women under the study, the blood samples were collected repeatedly in the first trimester (gestation age of 12 weeks or less) and early second trimester (during weeks 16-20 of gestation). In this study, we used the data from two repeated measures of hemoglobin ( $\mathrm{Hb})$, hematocrit (Hct), fasting blood sugar (FBS) and red blood cell count (RBC) in the described trimesters as the early predictors of GDM. 


\section{Statistical analysis}

In the first stage of data analysis, to compare the different characteristics of the women with and without GDM, we used the ordinary chi-square test and independent samples t-test. In addition, the repeated measures analysis of variance was utilized to compare the repeated levels of the described biomarkers in two trimesters between GDM and non-GDM groups. To achieve the main goal of the present study (evaluating the predictive power of longitudinal multiple biomarkers for early detection of GDM), we applied the method suggested by Roy which uses multivariate longitudinal data (biomarkers) for classifying sampling units into different subgroups (GDM or non-GDM) (29). In this context, we first use the following multivariate longitudinal random-effects model to assess the effect of different covariates on repeated biomarkers:

$$
\begin{gathered}
\boldsymbol{y}_{i}=\boldsymbol{X}_{i} \boldsymbol{\beta}+\boldsymbol{Z}_{i} \boldsymbol{b}_{i}+\boldsymbol{\varepsilon}_{i} \\
\boldsymbol{b}_{i} \sim N(\mathbf{0}, \boldsymbol{D}) \\
\boldsymbol{\varepsilon}_{i} \sim N\left(\mathbf{0}, \boldsymbol{\Omega}_{\boldsymbol{i}}\right)
\end{gathered}
$$

where $\boldsymbol{y}_{i} \boldsymbol{X}_{i} \boldsymbol{\beta}, \boldsymbol{b}_{i}, \boldsymbol{Z}_{i}$ and $\boldsymbol{\varepsilon}_{i}$ indicate the response variables, matrix of time-stationary (such as parity and mother educational level) and time-varying (such as Body Mass Index, time of measurement, systolic and diastolic blood pressure) covariates, vector of regression parameters, matrix of time-varying covariates, random term and random errors, respectively. In this model, $D$ and show the variance-covariance matrices of random terms and random errors, respectively.

In the modeling process, we considered four repeated biomarkers as the multivariate longitudinal response variables $\left(\boldsymbol{y}_{\mathrm{i}}\right)$. In addition, the Body Mass Index (BMI), systolic blood pressure (SBP), diastolic blood pressure (DBP), presence of GDM ( $1=$ non-GDM, 2=GDM) and time of measurement (1=first trimester, $2=$ second trimester) were considered as the model covariates $\left(\boldsymbol{X}_{\boldsymbol{i}}\right)$. After fitting this multivariate longitudinal model and estimating the parameters, we used the following classification rule for discriminating pregnant women in terms of being in non-GDM (population 1) or GDM (population 2) groups:

Allocate the ith woman to non-GDM group if

$$
\left(\boldsymbol{\mu}_{1 i}-\boldsymbol{\mu}_{2 i}\right)^{\prime}\left(\boldsymbol{Z}_{i} \boldsymbol{D} \boldsymbol{Z}_{i}^{\prime}+\operatorname{dim}\left(\boldsymbol{\Omega}_{i}\right)\right)^{-1}\left(\boldsymbol{y}_{i}-\frac{1}{2}\left(\left(\boldsymbol{\mu}_{1 i}+\boldsymbol{\mu}_{2 i}\right)\right) \geq \ln \left(\frac{\boldsymbol{\pi}_{2}}{\boldsymbol{\pi}_{1}}\right)\right.
$$

and to GDM group otherwise. In this equation, $\boldsymbol{I}_{1}$ and $\boldsymbol{I}_{2}$ indicate the proportion of population 1 and 2 (in our study, the proportion of GDM and non-GDM women), $\boldsymbol{\mu}_{\mathrm{i} 1}$ and $\boldsymbol{\mu}_{\mathrm{i} 2}$ show the mean response variables for th subject in group 1 and 2 (in our study, the mean values of biomarkers in GDM and nonGDM women). In addition, notation dim indicates the dimension of the matrix. 
In this discrimination technique, two different strategies were utilized to assess the power of RBC, $\mathrm{Hb}$, $\mathrm{HcT}$ and FBS for predicting GDM. At the first stage, we used the repeated measures data (biomarker level

in the first and second trimesters) from each biomarker to estimate the predictive performance of every single biomarker under the study (univariate strategy). In the next stage, the repeated measures data from all the described biomarkers were used in a multivariate framework to determine the predictive power indices of all biomarkers concurrently (multivariate strategy).

\section{Results}

In this prospective cohort study, the data from 600 pregnant women was analyzed. The mean (SD) age and BMI of the sample women was 27.28 (3.94) and 24.99 (4.37), respectively. More than one-fourth of women under the study had academic education.

Among the sample pregnant women, 49 cases (8.2\%) were diagnosed with GDM by the end of trimester 3. Table 1 shows the characteristics of these women by GDM. As we can see, the mean age of women with GDM was significantly higher than non-GDMs $(P=0.048)$. In the second trimester of pregnancy, the mean systolic and diastolic blood pressures were also significantly higher in GDM patients than nonGDM women $(P=0.005$ and $P=0.008$, respectively). 
Table 1

Comparing the demographic and clinical characteristics between two groups

\begin{tabular}{|c|c|c|c|c|}
\hline Characteristics & Category & Non-GDM $(n=551)$ & $\operatorname{GDM}(n=49)$ & $\mathrm{p}$ \\
\hline \multirow[t]{4}{*}{ Parity } & 0 & $1(0.2)^{*}$ & $0(0)$ & 0.689 \\
\hline & 1 & $260(47.2)$ & $28(57.1)$ & \\
\hline & 2 & $224(40.6)$ & 15(30.7) & \\
\hline & 3 & $66(12)$ & $6(12.2)$ & \\
\hline \multirow[t]{2}{*}{ Education } & Non-Academic & $399(72.4)$ & $38(77.6)$ & 0.816 \\
\hline & Academic & $152(27.6)$ & $11(22.4)$ & \\
\hline \multirow[t]{2}{*}{ Folic Acid use } & Yes & $530(96.2)$ & $47(95.9)$ & 0.925 \\
\hline & No & $21(3.8)$ & $2(4.1)$ & \\
\hline \multirow[t]{2}{*}{ Iron use } & Yes & $539(97.8)$ & $49(100)$ & 0.356 \\
\hline & No & $12(2.2)$ & $0(0)$ & \\
\hline \multirow[t]{2}{*}{ Calcium use } & Yes & $366(66.4)$ & $33(67.3)$ & 0.896 \\
\hline & No & 185(33.6) & $16(32.7)$ & \\
\hline \multirow[t]{2}{*}{ Vitamin use } & Yes & $432(78.4)$ & $42(85.7)$ & 0.229 \\
\hline & No & 119(21.6) & $7(14.3)$ & \\
\hline Age (years) & - & $27.19 \pm 3.93^{\star \star}$ & $28.35 \pm 3.89$ & 0.048 \\
\hline BMI (trimester 1) & - & $24.89 \pm 4.30$ & $26.09 \pm 5.02$ & 0.066 \\
\hline BMI (trimester 2) & - & $29.73 \pm 4.11$ & $30.90 \pm 4.45$ & 0.059 \\
\hline DBP (trimester 1) & - & $64.2 \pm 7.7$ & $63.47 \pm 8.05$ & 0.525 \\
\hline DBP (trimester 2) & - & $66.16 \pm 9.57$ & $70.01 \pm 10.60$ & 0.008 \\
\hline SBP (trimester 1) & - & $101.79 \pm 12.31$ & $101.73 \pm 10.78$ & 0.977 \\
\hline SBP (trimester 2) & - & $106.56 \pm 14.43$ & $112.55 \pm 13.62$ & 0.005 \\
\hline \multicolumn{5}{|l|}{ *No(\%) } \\
\hline$\star *-x \pm S D$ & & & & \\
\hline
\end{tabular}


Table 2

Comparing the mean trend of different markers in GDM and non-GDM patients

\begin{tabular}{|lllll|}
\hline Marker & Trimester & GDM & Non- GDM & P \\
\hline RBC $\left(\times 10^{12}\right)$ & 1 & $4.58 \pm 0.45$ & $4.39 \pm 5.01$ & 0.080 \\
& 2 & $4.19 \pm 0.41$ & $4.12 \pm 0.45$ & \\
\hline Hb (g/dL) & 1 & $13.22 \pm 1.17$ & $12.63 \pm 0.99$ & $<0.001$ \\
& 2 & $12.32 \pm 0.90$ & $11.89 \pm 0.97$ & \\
\hline Hct (\%) & 1 & $39.53 \pm 3.35$ & $37.55 \pm 3.54$ & $<0.001$ \\
& 2 & $36.87 \pm 2.54$ & $35.86 \pm 2.88$ & \\
\hline FBS (mg/dL) & 1 & $92.04 \pm 12.01$ & $83.19 \pm 11.75$ & $<0.001$ \\
& 2 & $96.96 \pm 12.74$ & $81.08 \pm 10.17$ & \\
\hline
\end{tabular}

Table 2 shows the obtained results from the repeated measures analysis of variance test for comparing the mean trend of four biomarkers (RBC, Hb, Hct and FBS) between women with and without GDM. In this context, we first assessed the interaction between group and time variables. Since none of the interactions were significant, we only reported the group effect in the last column of Table 2 to compare the mean trend of the biomarkers between two groups. From these results, one can conclude that the mean $\mathrm{Hb}$, Hct and FBS were significantly higher in GDM patients compared to non-GDMs $(P<0.001)$. At the same time, although the mean RBC was slightly higher in GDMs than non-GDM women, there was no significant difference between two groups $(P=0.080)$.

In the next step of data analysis, we used the ordinary univariate ROC curve analysis in addition to the explained multivariate longitudinal discriminant analysis to identify the predictive power of markers in early diagnosis of GDM. The estimated values of sensitivity, specificity and area under curve for these markers were presented in Table 3. As can be seen, when we use RBC, Hb, Hct and FBS as a single indicator for predicting GDM (univariate analysis), the estimated values of AUC seem to be quite unsatisfactory (ranging from $61 \%$ for RBC to $68 \%$ for FBS). On the other hand, when we simultaneously utilized these biomarkers for predicting GDM (multivariate analysis), more acceptable estimates could be obtained for the sensitivity, specificity and subsequently AUC indices. In other words, concurrent use of these biomarkers for early prediction of GDM would results in an estimated sensitivity of $87 \%$, specificity of $70 \%$ and $A U C$ of $83 \%$. Figure 1 displays the estimated ROC curves from univariate and multivariate analysis of the biomarkers. 
Table 3

Estimated predictive power indices using univariate and multivariate analysis of different markers in early diagnosis of GDM

\begin{tabular}{|lllll|}
\hline Marker & Sensitivity & Specificity & AUC & $95 \% \mathrm{Cl}$ for AUC \\
\hline RBC & 60 & 54 & 61 & $50-71$ \\
$\mathrm{Hb}$ & 67 & 62 & 67 & $61-73$ \\
$\mathrm{HCT}$ & 62 & 61 & 65 & $59-71$ \\
FBS & 68 & 60 & 68 & $60-75$ \\
Multivariate & 87 & 70 & 83 & $76-90$ \\
\hline
\end{tabular}

\section{Discussion}

In the past few decades, the global burden of type II diabetes Mellitus seems to be increasing due to population aging and unhealthy lifestyle including bad eating habits and insufficient physical activity. For the same reasons, the prevalence of GDM has globally increased in some parts of the world including developing countries (11). The results of some epidemiologic studies showed about one-third increase in the global prevalence of GDM within the past decades $(2,3)$. The increasing pattern in prevalence of GDM consequently causes a rising trend in economic burden of this disease. According to a study in Italy, the antenatal outpatients cost per case in GDM patients was estimated about $61.5 \%$ higher than normal pregnancies (30). A modeling study in Chinese population who gave birth in 2015 revealed that GDM nearly doubles the average cost of pregnancy in this country (15). On this basis, early diagnosis of GDM might help the local, regional and national health policy makers to reduce the burden of this disease in their areas.

In our data analysis process, we first compared the mean repeated measures of RBC, $\mathrm{Hb}$, Hct and FBS between GDM and non-GDM groups. The obtained results revealed that the mean levels of these four biomarkers were higher in women with GDM than non-GDMs (all p-values were significant except for RBC which showed a borderline significancy). The relationship between GDM and some of these biomarkers has been previously evaluated by other researchers in a number of cross-sectional or cohort studies. In a prospective study conducted by Wu et al., they compared some clinical characteristics of women with and without GDM during weeks 12-16 of gestation. Similar to our results, they reported significant higher mean levels of $\mathrm{RBC}, \mathrm{Hb}$, Hct and FBS in women with GDM compared to healthy pregnancies. Their findings also showed higher levels of BMI, SBP, DBP, platelet count, glycated hemoglobin, total cholesterol, triglyceride, HDL and folic acid as well as lower levels of glacyted albumin and vitamin B12 in GDMs compared to non-GDMs (28). In a hospital-based retrospective study among more than 21000 Chinese pregnant women, the results confirmed the relationship between increased $\mathrm{Hb}$ levels during early pregnancy and development of GDM. Their findings resulted in an odds ratio of 1.27 for developing GDM in women with increased levels of $\mathrm{Hb}$ compared to other pregnant women (27). In another cohort study among 600 Iranian pregnant women, estimated relative risks of 2.12 and 1.47 were reported for 
developing GDM in women with high $\mathrm{Hb}$ levels compared to those with normal $\mathrm{Hb}$, respectively in the second and third trimesters of pregnancy (31). Regarding these findings, it can be concluded that all of the described biomarkers could be used as potential diagnostic indicators for early detection of GDM.

In the present study, our main objective was to investigate the diagnostic accuracy of some available indicators in routine blood test for early detection of GDM. Our findings showed that using the repeated measures of RBC, $\mathrm{Hb}, \mathrm{Hct}$ and FBS in the first and early second trimesters of pregnancy leads to a sensitivity of $87 \%$, specificity of $70 \%$ and AUC of $83 \%$ for early prediction of GDM. In other words, the estimated AUC tells us that more than four-fifth of pregnant women could be correctly classified into GDM or non-GDM group using the multiple repeated measures of the described biomarkers. As mentioned in the introduction section, a number of studies have previously examined the diagnostic power of different biomarkers for predicting the development of GDM. In a case-control study on 12 GDM patients and 60 controls, the researchers used the logistic regression model and concluded that combination of Adipo, PAPP-A and BMl yields a detection rate of $72.7 \%$ at a false positive rate of $10 \%$ for detecting GDM (21). In another study on 523 pregnant women, Amini et al. applied more complicated statistical modeling approach (Bayesian latent class models) to assess diagnostic accuracy of multiple markers for early detection of GDM. Using the $\beta$-hCG, uE3 and AFP as the multiple biomarkers, they reached to a sensitivity of $94 \%$, specificity of $86 \%$ and AUC of $92 \%$ (22). In another study by TenenbaumGavish et al., the researchers used obesity, placental and inflammatory biomarkers for prediction of GDM in the first trimester of pregnancy. Their results showed an AUC of $95 \%$ with detection rate of $89 \%$ at $10 \%$ false positive rate for predicting GDM in obese expectant women using the combination of high BMI, insulin, soluble CD163 and TNFa results. They also reported an AUC of $94 \%$ with detection rate of $83 \%$ at $10 \%$ false positive rate for prediction GDM in non-obese women using the combination of soluble CD163, TNFa, PP13 and PAPP-A (23). Zhao et al. used the data from 876 singleton pregnancies to assess the predictive power of early second trimester maternal serum markers in prediction of GDM. At a specificity level of $80 \%$, their findings showed detection rate of $94.9 \%$ for pentraxin 3 (PTX3), $92.3 \%$ for PP13, $94.9 \%$ for soluble fms-like tyrosine kinase-1 (sFLt-1), $92.5 \%$ for myostatin and $92.3 \%$ for follistatin (FST) (25). Wu et al evaluated the accuracy power of glycated hemoglobin A1c $(\mathrm{HbA} 1 \mathrm{c})$ alone and in combination with HcT for screening GDM patients between 12-16 gestational weeks. They concluded that HbA1c as a single biomarker had no acceptable predictive power for detecting GDM (with an AUC of 56.3\%) while the combination of $\mathrm{HbA} 1 \mathrm{c}$ and HcT might be a useful screening test for GDM (with an AUC of $60.4 \%$ ) (28). In a recent research in this field, Lorenzo-Almorós et al. reviewed large number of predictive and diagnostic biomarkers for GDM including protein and genetic biomarkers they concluded that reduction in liverderived sex hormone blinding globulin (SHBG) and adiponectin, in combination with increased levels of RBP4, afamin, ficlon-3 and some specific MiRs could be a reliable measure for prediction GDM (26). In conclusion, reviewing the published works in this field indicates that there is no consensus among researchers regarding the best feasible framework for early prediction of GDM. It seems that more comprehensive studies with higher sample sizes and wider range of biomarkers should be conducted to identify more reliable and globally practicable standard for the diagnosis of GDM in the earlier stages of pregnancy. 
Similar to other studies in this field, our study has some limitations and strengths. In our opinion, applying a complex statistical method which allows us to concurrently include the information from the longitudinal multiple biomarkers (Hct, $\mathrm{Hb}$, FBS and RBC levels in the first and early second trimesters of pregnancy) in a single predictive tool was the most important strength point of the current study. By using this approach, we presented a feasible framework for early prediction of GDM via inexpensive accessible parameters from the routine $\mathrm{CBC}$ tests in different trimesters of pregnancy. In addition, analyzing the data from a relatively adequate sample size, at least in comparison with most of the related studies in this field (assessing the predictive power of different biomarkers for early detection of GDM), could be considered as another strength point of the present work. On the other hand, using a non-random sampling technique (consecutive sampling method) for selecting the study sample might be thought as the main limitation of our study. This limitation may call into question the generalization of some inferences in our work. Regarding this, conducting more comprehensive studies using random sampling approaches (for instance, multi-stage cluster sampling technique) and more generalizable sample sizes might be needed to evaluate the predictive power of these biomarkers for early detection of GDM more accurately.

\section{Conclusion}

In this study, we employed more advanced statistical methods to suggest a framework for early detection of GDM by using common parameters in the routine CBC tests. The estimated indices of predictive accuracy for the described method showed that about $80 \%$ of pregnant women could be properly categorized into GDM and non-GDM groups using our suggested framework. Although some researchers have previously introduced a number of biomarkers with higher predictive power for early detection of GDM, some of these biomarkers are costly or hard to access for all pregnant women in the majority of poor or low-income countries. Thus, there is an urgent need in the future works to focus on more popular and accessible indicators for early prediction of GDM, especially in countries with lower levels of health resources.

\section{Abbreviations}

Gestational Diabetes Mellitus: GDM

Complete Blood Count: CBC

Hemoglobin: $\mathrm{Hb}$

Hematocrit: Hct

Fasting Blood Sugar: FBS

Red Blood Cell Count: RBC 
Area Under the Curve: AUC

International Diabetes Federation: IDF

American Diabetes Association: ADA

World Health Organization: WHO

Beta-human Chorionic Gonadotropin: $\beta$-hCG

Tumor-Necrosis Factor Alpha: TNFa

Unconjugated Estriol: uE3

Alfa-Fetoprotein: AFP

Pregnancy-Associated Plasma Protein A: PAPP-A

Placental Protein 13: PP13

Adiponectin: Adipo

Glucose Load Test: GLT

Oral Glucose Tolerance Test: OGTT

Body Mass Index: BMI

Systolic Blood Pressure: SBP

Pentraxin 3: PTX3

Soluble Fms-Like Tyrosine Kinase-1: sFLt-1

Follistatin: FST

Glycated Hemoglobin A1c: HbA1c

Sex Hormone Blinding Globulin: SHBG

\section{Declarations}

\section{Acknowledgements}

This article is part of a PhD dissertation in the field of biostatistics approved by the School of Allied Medical Sciences, Shahid Beheshti University of Medical Sciences, Tehran, Iran. The authors would like to 
express their gratitude to our colleagues in faculties of Allied Medical Sciences and Nursing and Midwifery for the facilitation of the process to conduct this study.

\section{Authors' contributions}

ESE proposed the statistical idea of the research, analyzed the data and wrote the initial draft of the manuscript. MN participated in analyzing the data and drafting the results and discussion sections. NK was the supervisor of the clinical part of the study. NSA proposed the main idea of the clinical part of the research and gathered the data. MS and MK participated in writing the initial draft of the manuscript and revised the final version. FZ was the supervisor of the statistical part of the research and participated in writing the initial draft of the manuscript and revised the final version. All authors read and approved the final manuscript.

\section{Funding}

No financial support was received for this research.

\section{Availability of data and materials}

The dataset analyzed in this study might be available from the corresponding author on reasonable request.

\section{Ethics approval and consent to participate}

The ethical aspects of the study was approved by the Ethics Committee of Shahid Beheshti University of Medical Sciences (Ethics Code: IR.SBMU.RETECH.REC.1397.823). Agreement to participate and a signed consent form were obtained from all pregnant women before data collection. In addition, all methods were carried out in accordance with relevant guidelines and regulations under Ethics approval and consent to participate.

\section{Consent for publication}

Not applicable

Competing interests The authors declare that they have no competing interests.

\section{Author details}

1,6Department of Biostatistics, School of Allied Medical Sciences, Shahid Beheshti University of Medical Sciences, Tehran, Iran. ${ }^{2}$ Department of Basic Sciences, Faculty of Nursing and Midwifery, Shahid Beheshti University of Medical Sciences, Tehran, Iran. ${ }^{3}$ Department of Midwifery and Reproductive Health Research Center, Faculty of Nursing and Midwifery, Shahid Beheshti University of Medical Sciences, Tehran, Iran. ${ }^{4}$ Department of Midwifery, Ardabil Branch, Islamic Azad University, Ardabil, Iran. ${ }^{5}$ Health Management and Economics Research Center and Department of Biostatistics, School of Public Health, 
Iran University of Medical Sciences, Tehran, Iran. ${ }^{7}$ Proteomics Research Center and Department of Biostatistics, School of Allied Medical Sciences, Shahid Beheshti University of Medical Sciences, Tehran, Iran.

\section{References}

1. Metzger BE, Coustan DR, Committee O. Summary and recommendations of the fourth international workshop-conference on gestational diabetes mellitus.Diabetes Care. 1998;21:B161-7.

2. Ferrara A. Increasing prevalence of gestational diabetes mellitus: a public health perspective.Diabetes Care. 2007;30 Suppl 2:S141-6. doi:10.2337/dc07-s206.

3. Anna V, van der Ploeg HP, Cheung NW, Huxley RR, Bauman AE. Sociodemographic correlates of the increasing trend in prevalence of gestational diabetes mellitus in a large population of women between 1995 and 2005.Diabetes Care. 2008;31(12):2288-93. doi:10.2337/dc08-1038.

4. Wendland EM, Torloni MR, Falavigna M, Trujillo J, Dode MA, Campos MA, et al. Gestational diabetes and pregnancy outcomes-a systematic review of the World Health Organization (WHO) and the International Association of Diabetes in Pregnancy study groups (IADPSG) diagnostic criteria.BMC Pregnancy Childbirth. 2012;12(1):23.

5. HAPO Study Cooperative Research Group. Hyperglycemia and adverse pregnancy outcomes. N Engl J Med. 2008;358(19):1991-2002. doi: 10.1056/NEJMoa0707943.

6. Bellamy L, Casas J-P, Hingorani AD, Williams D. Type 2 diabetes mellitus after gestational diabetes: a systematic review and meta-analysis.Lancet. 2009;373(9677):1773-9.

7. Retnakaran R, Qi Y, Connelly PW, Sermer M, Zinman B, Hanley AJ. Glucose intolerance in pregnancy and postpartum risk of metabolic syndrome in young women.J Clin Endocrinol Metab. 2010;95(2):670-7.

8. Marchetti D, Carrozzino D, Fraticelli F, Fulcheri M, Vitacolonna E. Quality of life in women with gestational diabetes mellitus: a systematic review.J Diabetes Res. 2017;2017:12. Article ID 7058082.

9. Langer O, Yogev Y, Most O, Xenakis EM. Gestational diabetes: the consequences of not treating. Am J Obstet Gynecol. 2005;192(4):989-97.

10. Clausen TD, Mathiesen ER, Hansen T, Pedersen O, Jensen DM, Lauenborg J, et al. High prevalence of type 2 diabetes and pre-diabetes in adult offspring of women with gestational diabetes mellitus or type 1 diabetes: the role of intrauterine hyperglycemia.Diabetes Care. 2008;31(2):340-6.

11. ZhuY, Zhang C. Prevalence of gestational diabetes and risk of progression to type 2 Diabetes: a Global Perspective.Curr Diab Rep. 2016;16(1):7. doi:10.1007/s11892-015-0699-x.

12. Seshiah V, Das A, Balaji V, Joshi SR, Parikh M, Gupta S. Gestational diabetes mellitus-guidelines.JAPI - DIPSI Guidelines. 2006;54:622.

13. International Diabetes Federation. IDF Diabetes Atlas, 6th edn. Brussels, Belgium: International Diabetes Federation, 2013 p.44. 
14. Dall Tm, Yang W, GillespieK, Mocarski M, Byrne E, Cintina I, et al. The Economic Burden of Elevated Blood Glucose Levels in 2017: Diagnosed and Undiagnosed Diabetes, Gestational Diabetes Mellitus, and Prediabetes.Diabetes Care 2019;42:1661-1668. doi:10.2337/dc18-1226.

15. Xu T, Dainelli L, Yu K, Ma L, Zolezzi IS, Detzel P, et al. The short-term health and economic burden of gestational diabetes mellitus in China: a modelling study. BMJ Open. 2017;7:e018893. doi:10.1136/bmjopen-2017-018893.

16. American Diabetes Association. Standards of medical care in diabetes-2011. Diabetes Care. 2011;34:S11-61. doi:10.2337/dc11-S011.

17. American Diabetes Association. Standards of medical care in diabetes-2014.Diabetes Care. 2014;37 Suppl 1:S14-80. doi:10.2337/dc14-S014.

18. World Health Organization/International Diabetes Federation. Definition and diagnosis of diabetes mellitus and intermediate hyperglycemia: report of a WHO/IDF consultation. Geneva: WHO; 2006.

19. World Health Organization. Diagnostic criteria and classification of hyperglycaemia first detected in pregnancy. Geneva: WHO; 2013.

20. Lee KW, Ching SM, Ramachandran V, Yee A, Hoo FK, Chia YC, et al. Prevalence and risk factors of gestational diabetes mellitus in Asia: a systematic review and meta-analysis. BMC Pregnancy Childbirth. 2018;18(1):494. doi: 10.1186/s12884-018-2131-4.

21. Farina A, Eklund E, Bernabini D, Paladino M, Righetti F, Monti G, et al. A First-Trimester Biomarker Panel for Predicting the Development of Gestational Diabetes.Reprod Sci. 2017;24(6):954-959. doi: $10.1177 / 1933719116675057$.

22. Amini M, Kazemnejad A, Zayeri F, Montazeri A, Rasekhi A, Amirian A, et al. Diagnostic accuracy of maternal serum multiple marker screening for early detection of gestational diabetes mellitus in the absence of a gold standard test.BMC Pregnancy Childbirth. 2020;20(1):375. doi: 10.1186/s12884020-03068-7.

23. Tenenbaum-Gavish K, Sharabi-Nov A, Binyamin D, Møller HJ, Danon D, Rothman L, et al. First trimester biomarkers for prediction of gestational diabetes mellitus. 2020 Nov;101:80-89. doi: 10.1016/j.placenta.2020.08.020.

24. Alanen J, Appelblom H, Korpimaki T, Kouru H, Sairanen M, Gissler M, et al. Glycosylated fibronectin as a first trimester marker for gestational diabetes.Arch Gynecol Obstet. 2020;302(4):853-860. doi: 10.1007/s00404-020-05670-8.

25. Zhao B, Han X, Meng Q, Luo Q. Early second trimester maternal serum markers in the prediction of gestational diabetes mellitus.J Diabetes Investig. 2018;9(4):967-974. doi: 10.1111/jdi.12798.

26. Lorenzo-Almorós A, Hang T, Peiró C, Soriano-Guillén L, Egido J, Tuñón J, et al. Predictive and diagnostic biomarkers for gestational diabetes and its associated metabolic and cardiovascular diseases.Cardiovasc Diabetol. 2019;18(1):140. doi: 10.1186/s12933-019-0935-9.

27. Wang C, Lin L, Su R, Zhu W, Wei Y, Yan J, et al. Hemoglobin levels during the first trimester of pregnancy are associated with the risk of gestational diabetes mellitus, preeclampsia and preterm 
birth in Chinese women: a retrospective study.BMC Pregnancy Childbirth. 2018;18(1):263. doi: 10.1186/s12884-018-1800-7.

28. Wu K, Cheng Y, Li T, Ma Z, Liu J, Zhang Q, et al. The utility of HbA1c combined with haematocrit for early screening of gestational diabetes mellitus.Diabetol Metab Syndr. 2018, 10:14. doi: 10.1186/s13098-018-0314-9.

29. Roy A. A new classification rule for incomplete doubly multivariate data using mixed effects model with performance comparisons on the imputed data.Statist Med. 2006; 25:1715-1728. doi: $10.1002 / \operatorname{sim} .2320$.

30. Meregaglia M, Dainelli L, Banks H, Benedetto C, Detzel P, Fattore G. The short-term economic burden of gestational diabetes mellitus in Italy. BMC Pregnancy Childbirth. 2018;18(1):58. doi: 10.1186/s12884-018-1689-1.

31. Rostami Mogaddam M, Safavi Ardebili N, Kariman N. The Relation Between the Incidence Rate of Second and Third Trimester Hemoglobin and the Incidence of Preeclampsia and Gestational Diabetes: A Cohort Study.Crescent J Med Biol Sci. 2019;6(1):85-90.

\section{Figures}




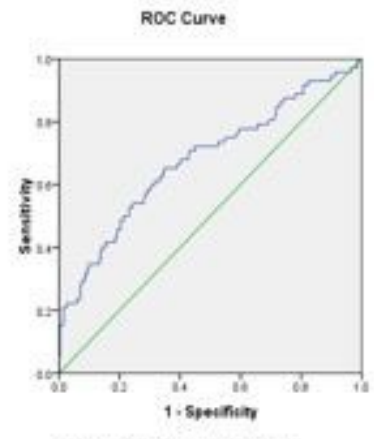

(a)

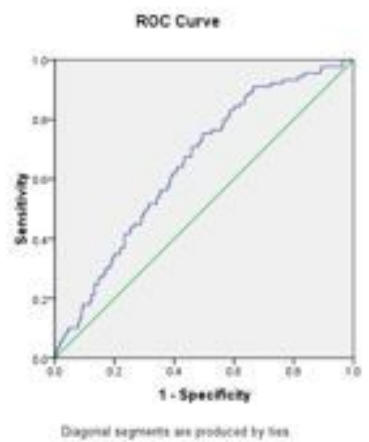

(c)
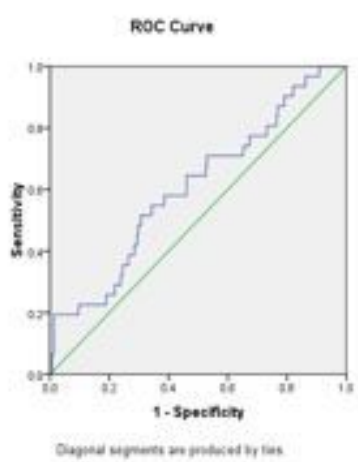

(b)

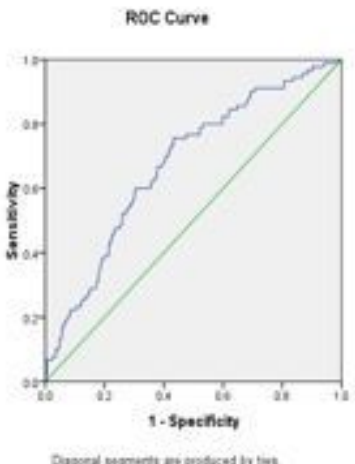

(d)

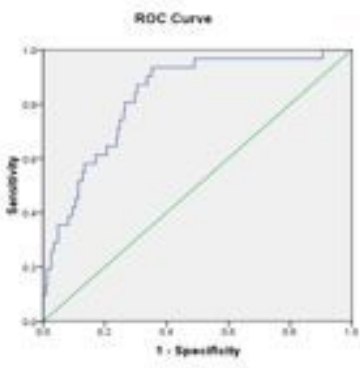

(e)

\section{Figure 1}

Roc curves for univariate and multivariate analysis of markers; a: RBC, b: FBS, c: Hct, d: Hb, e: Multivariate 